\title{
Le marqueur discursif bon : ses fonctions et sa position dans le français parlé
}

\author{
Joy P. G. Peltier ${ }^{1, *}$ et Diana L. Ranson ${ }^{2}$ \\ ${ }^{1}$ Department of Linguistics, University of Michigan, Ann Arbor, MI 48109, USA \\ ${ }^{2}$ Department of Romance Languages, University of Georgia, Athens, GA 30602, USA
}

\begin{abstract}
Résumé. À travers l'examen de 227 occurrences du marqueur discursif bon dans un corpus de français parlé, cette étude présente une nouvelle vue d'ensemble de ses fonctions pragmatiques. Nous proposons que bon est essentiellement un marqueur de réorientation et d'acceptation. La réorientation peut être de nature textuelle quand bon marque l'introduction ou la reprise des unités de discours, ou la formulation ou la reformulation des idées du locuteur. D'autre part, cette réorientation est attitudinale quand bon marque le contraste, la résignation, et l'atténuation du message du locuteur. Une analyse de la place de bon dans le tour de parole et dans la phrase n'a pas discerné de patron net entre ses fonctions et sa position. Le locuteur insère bon dans les moments du discours où il veut signaler son acceptation du discours précédent en même temps qu'il indique une réorientation majeure ou mineure dans la structure de la conversation. L'examen des séquences de bon avec d'autres éléments qui remplissent des fonctions similaires nous a permis d'évaluer si sa fonction d'acceptation ou de réorientation était mise en avant dans un certain contexte.
\end{abstract}

\begin{abstract}
The discourse marker bon: its functions and position in Spoken French. Through an analysis of 227 tokens of the discourse marker bon in a corpus of Spoken French, this study presents a new organization of its pragmatic functions. We propose that bon is essentially a marker of reorientation and acceptance. Reorientation can be of a textual nature when bon marks the introduction or the resumption of discourse units, or the formulation or reformulation of the speaker's ideas. On the other hand, this reorientation is attitudinal when it signals contrast, resignation or a softening of the speaker's message. An analysis of the place of bon within the speech turn and the sentence did not reveal any clear-cut pattern between its functions and position. Speakers insert bon at points in the discourse where they want to signal acceptance of the previous discourse while at the same time indicating a major or minor reorientation in the structure of the conversation. The analysis of sequences of bon with other elements that fulfill similar functions made it possible for us to evaluate whether its acceptance or reorientation function was highlighted in a certain context.
\end{abstract}

* Corresponding author: joypel@umich.edu 


\section{La multifonctionnalité du marqueur discursif bon}

Le marqueur discursif bon, dérivé de l'adjectif bon et attesté depuis le $\mathrm{XVI}^{\mathrm{e}}$ siècle (Waltereit 2007 : 99), remplit aujourd'hui une variété de fonctions pragmatiques bien éloignées de la simple évaluation positive qu'exprimait l'adjectif. Certaines de ses fonctions, celles qui indiquent la structure du discours, se qualifient de textuelles. Les marqueurs discursifs avec ces fonctions «segmentent le discours oral comme la ponctuation et les marques de paragraphe segmentent les textes écrits »(Crible 2017: 77). Dans (1), par exemple, le locuteur $(\mathrm{M})$ dit bon pour marquer le début de sa réponse sur un nouveau thème proposé par son interlocutrice (D).

1) D : C'était quelle sorte de cuisine?

$\mathrm{M}$ : Bon c'est la cuisine où il y a beaucoup de poissons, c'est au bord de mer. (JHS24, \#R11-7) 1

D'autres fonctions de bon se décrivent comme attitudinales puisqu'elles expriment l'attitude du locuteur envers le sujet de la conversation ou envers son interlocuteur. Dans (2) le même locuteur se sert de bon pour exprimer son attitude sur son travail dans les grandes surfaces.

2) $\mathrm{M}:$ c'étaient des gardes de nuit dans les campings, ça c'est très sympathique, parce que vous rencontrez beaucoup de monde la nuit [...] j'ai fait aussi les grandes surfaces, $\underline{\text { bon }}$ ça c'était un peu moins bien, c'est bien, c'est des postes très longs, huit neuf dix heures d'affilé à rester debout sans bouger. (JHS24, \#R11-7)

Les marqueurs discursifs (désormais MD) sont caractérisés par leurs formes courtes et invariables (Heine 2013 : 1209), leur rôle dans la connectivité structurelle entre les segments du discours (Hansen 1997 : 160-161), leur optionalité syntaxique, leur manque de contenu propositionnel, et leur multifonctionnalité (Crible 2017 : 44-47, 58). C'est cette multifonctionnalité qui pose un des plus grands défis pour une analyse cohérente et compréhensive des fonctions des MD, tels que bon (Frank-Job 2005 : 395). Plusieurs auteurs ont observé que bon comme MD conserve des traces de son sens adjectival quand il fonctionne comme un "évaluateur de discours» (Lefeuvre 2011a : 224, voir aussi Winther 1985 : 82-83, Hansen 1998a : 253-54, Waltereit $2007: 100$, Skattum 2012 : 20911). Dostie (2009 : 203) souligne en plus que c'est la fonction d'exprimer une évaluation positive qui fait que bon soit susceptible de développer des emplois comme MD. D'autres auteurs, qui constituent la majorité, insistent sur ses fonctions textuelles. Barnes (1995), par exemple, le voit comme un marqueur de transition, tandis que Hansen (1998a : 25356) maintient qu'il signale un événement discursif indésirable. Lefeuvre (2011a : 226), qui cite Brémond (2002), le décrit comme « une charnière » qui articule " deux unités syntaxiques prédicatives ». Pour Beeching (2007: 81) bon sert à ponctuer les étapes successives d'une narration, tandis que pour Jayez (2004) bon est un " mot de la fin » employé par un locuteur qui veut que le discours soit terminé. Parmi les fonctions attitudinales de bon, plusieurs auteurs ont dit qu'il sert à adoucir le message du locuteur en exprimant la réserve (Hansen 1998b : 245-46, Beeching $2007: 81$ ) ou l'atténuation (Beeching 2011 : 95, Skattum 2012 : 209-10).

Face à la multifonctionnalité de bon, cet article propose de recenser les fonctions de toutes ses occurrences dans un corpus de français parlé pour arriver à une nouvelle vue d'ensemble de ses emplois. Nous proposons que les instructions sémantico-pragmatiques de bon sont surtout de signaler à l'interlocuteur une réorientation, qui peut être textuelle ou attitudinale ou les deux à la fois. En signalant une réorientation, bon peut en même temps indiquer une reconnaissance ou acceptation du discours précédent et un 
(re)démarrage des propos de l'interlocuteur. Dans (1) ci-dessus, par exemple, le locuteur dit bon pour marquer la réorientation vers sa réponse tout en acceptant la question de son interlocutrice. En plus de proposer et exemplifier les différentes fonctions de bon dans un corpus, cette étude calcule la fréquence de ces fonctions pour identifier celles qui sont les plus répandues et pour déterminer s'il existe une corrélation entre la place de bon dans le discours et sa fonction. En outre, pour mieux cerner la contribution de bon, nous considérons aussi le rôle de bon vis-à-vis des autres éléments qui le précèdent ou le suivent comme la conjonction mais, la particule d'hésitation euh, l'adverbe puis, et les mots d'acceptation oui et voilà. L'organisation de l'article correspond à ces buts. Après la description du corpus et des méthodes de classification dans la section 2, la section 3 présente les fonctions pragmatiques de bon. La section 4 présente leur fréquence dans le corpus et leur répartition selon la position dans le discours. La section 5 considère la fonction de bon dans ses combinaisons avec d'autres éléments. La conclusion dans la section 6 résume les résultats de l'étude et propose des pistes pour de futures recherches.

\section{Corpus et méthodes}

Pour identifier les fonctions de bon comme MD, il était nécessaire dans un premier temps de recueillir toutes les occurrences du MD bon dans un corpus de français parlé. L'échantillon analysé pour la présente étude comprend 9 heures et demie de conversations, sur un total de 25 heures, enregistrées à Montpellier en 2005 et à Rognes en 2006 entre la deuxième auteure et 22 femmes et 15 hommes. Parmi les 22 locutrices, 14 viennent du sud de la France et 8 du nord. Cinq locutrices du sud et 4 locutrices du nord sont jeunes (entre 22 et 35 ans) et 9 du sud et 4 du nord sont plus âgées (entre 40 et 80 ans). Seulement 4 locutrices âgées du sud n'ont pas le baccalauréat. Parmi les 15 locuteurs masculins, 14 sont originaires du sud, dont 7 jeunes et 7 plus âgés, contre seulement 1 jeune homme du nord avec le baccalauréat. Deux jeunes locuteurs du sud et 4 locuteurs plus âgés n'ont pas le baccalauréat. L'identifiant de chaque locuteur indique son âge ( $\mathrm{J}=$ jeune ou $\mathrm{A}=\mathrm{a} g e ́)$, son sexe ( $\mathrm{F}=$ femme ou $\mathrm{H}=$ homme), sa région d'origine ( $\mathrm{S}=$ sud ou $\mathrm{N}=$ nord) et son âge ; par exemple, JFN25 est une jeune femme du nord âgée de 25 ans. Nous présentons ces renseignements dans le souci de bien décrire nos locuteurs quoique leurs caractéristiques sociales ne figurent pas dans les résultats. Le but de cette étude est d'identifier les fonctions pragmatiques de bon dans un échantillon de français parlé quelles que soient les caractéristiques des locuteurs.

Dans l'identification des occurrences du MD bon dans notre corpus, nous avons pris soin d'écarter les occurrences de son double adjectival. Cette distinction s'est basée surtout sur des critères syntaxiques. L'adjectif bon apparait dans le même syntagme nominal que le substantif masculin qu'il modifie ou dans le prédicat tandis que le MD bon ne modifie aucun substantif et jouit de la liberté syntaxique d'apparaître en début, milieu, ou fin de phrase. En plus d'exclure les occurrences de l'adjectif bon, nous avons dû écarter un exemple de bon puisque la locutrice a arrêté sa phrase sans fournir le contexte nécessaire pour sa classification : Bon on serait sur ... (JFS22, \#R13-12).

Après avoir repéré les occurrences de bon dans notre corpus, nous sommes passées à leur classification. Pour les fonctions textuelles, nous avons tenu compte du contexte de chaque occurrence de bon pour déterminer la relation entre les propos avant et après bon. Pour les fonctions attitudinales nous avons dû nous appuyer sur notre compréhension de l'attitude du locuteur et son rapport avec ses interlocuteurs, une tâche facilitée par le rôle de la deuxième auteure comme interlocutrice dans ces conversations. Chacune des auteures a fait ses classifications indépendamment et ensuite nous les avons comparées et avons résolu ensemble les cas divergents. Les fonctions de bon présentées dans les travaux consultés nous ont servi de point de départ pour nos propres classifications, mais nous avons dû les modifier pour bien classer les occurrences de bon dans notre corpus. Il convient de signaler aussi que nous avons déterminé les fonctions de bon dans un premier 
temps sans considérer leur place dans la phrase ou le tour de parole, à l'encontre des autres auteurs qui ont considéré les fonctions de bon selon sa place dans la chaîne parlée (Winther 1985 : 85-89, Hansen 1998b : 234-247, Skattum 2012 : 212). Après avoir identifié les fonctions de bon, nous avons classé les occurrences selon leur position pour dégager des corrélations possibles entre la position et la fonction.

Il est important de mentionner que plusieurs occurrences de bon exprimaient une fonction qui était textuelle et attitudinale à la fois. Par exemple, dans (3) la locutrice se sert de et bon pour introduire une parenthèse, qui a la fonction textuelle de « supplément », en même temps qu'elle souligne un contraste entre sa critique du film et son approbation de son emploi dans la salle de classe. Nous avons choisi de classer ces exemples selon leur fonction textuelle dans les résultats quantitatifs de cette étude dans la Section 3 tout en reconnaissant la multifonctionnalité de bon dans ces exemples.

\section{3) F : Parce que, c'est, les jeunes de banlieue contre les policiers, c'est quand même ça. [...] \\ F : Donc, c'est vraiment le stéréotype quand même. Et bon, c'est pas mal à traiter, et l'histoire est bien. (JHS25, \#7-4)}

Une autre illustration de la coexistence d'une fonction textuelle et une fonction attitudinale se trouve dans l'exemple (4). Ici, le locuteur dit bon pour indiquer le commencement de la reformulation de son énoncé, mais en même temps il marque un contraste entre la disponibilité des postes à Montpellier en général et le nombre de débouchés dans le secteur scientifique.

4) D : est-ce qu'il y a de bons débouchés ou, comment ça marche?

$[\ldots]$

$\mathrm{M}:$ ben moi ça fait euh bien ça fait quatre ans que, je je tire les ficelles

$[\ldots]$ pour essayer de, non bon euh, nous en sciences euh on n'est pas trop à plaindre, il y a quand même des postes... (JHS28, \#8-7)

\section{Les fonctions pragmatiques de bon}

\subsection{Vue d'ensemble des fonctions pragmatiques de bon}

L'analyse des fonctions de bon dans notre corpus nous a portées à proposer une nouvelle organisation plus cohérente et plus complète pour les fonctions textuelles et attitudinales de bon dans le but de mieux saisir son rôle fondamental de marqueur de réorientation et d'acceptation. En faisant des distinctions fines entre les fonctions textuelles, nous avons voulu résumer les fonctions de bon proposées par les études antérieures et décrire les sortes de réorientations textuelles indiquées par bon. Le Tableau 1 ci-dessous montre que les études antérieures sur le MD bon prises ensemble ont identifié toutes les fonctions textuelles et attitudinales que nous avons trouvées pour bon dans notre corpus, à trois exceptions près, notamment le résultat, l'élément additif, et la résignation. Pourtant, aucune étude antérieure n'a identifié toutes ces fonctions.

La nouvelle vue d'ensemble des fonctions de bon propose neuf fonctions textuelles et deux fonctions attitudinales. Parmi les fonctions textuelles, le nouveau thème, la prise de thème, et la nouvelle voix sont classées sous la catégorie générale d'ouverture. Sous la rubrique de continuation sont regroupées quatre fonctions, notamment le sous-thème, le résultat, le supplément, et l'élément additif. Les deux dernières fonctions textuelles sont la reprise de thème et la formulation. Nous proposons aussi deux fonctions attitudinales, que nous avons pu quantifier, qui sont le contraste et la résignation. 


\subsection{Fonctions textuelles d'ouverture}

Cette section présente une explication et illustration de chacune des fonctions pragmatiques de bon présentées dans le Tableau 1 et leur comparaison avec les fonctions proposées dans les travaux consultés.

\subsubsection{Nouveau thème}

Les trois fonctions textuelles de bon qui servent à introduire un nouveau sujet de conversation sont classées comme des fonctions d'ouverture. Nous attribuons la fonction de «nouveau thème » à bon lorsque le locuteur s'en sert pour proposer un nouveau sujet de conversation. Une fonction similaire a été proposée aussi pour les MD voilà (Haileselassie 2015 : 76, 93), alors (Hansen 1997: 181), et mais en français et but en anglais (Dajko et Carmichael 2014 : 166). Dans (5), par exemple, la locutrice se sert de bon pour marquer le début de ses propos sur la variété de langue enseignée dans la salle de classe, un nouveau thème par rapport au thème précédent sur les variétés régionales d'une langue.

5) F : en Angleterre il y a des variétés d'accent infinies

$\mathrm{D}$ : oui, oui beaucoup hein

$\mathrm{F}:$ c'est pareil donc, c'est ça qui est très important

$\mathrm{E}:$ c'est une autre langue

$\mathrm{F}: \underline{\text { Bon }}$ il est sûr que quand vous enseignez une langue il faut $[. .$. qu'il y ait un corpus euh euh qui soit généralement accepté. Vous ne pouvez pas aller dans les régionalismes. (AFN65, \#15-4)

Tableau 1. Les fonctions de bon et leurs mentions dans la littérature

\begin{tabular}{|c|c|}
\hline \multicolumn{2}{|c|}{ Fonctions textuelles } \\
\hline \multicolumn{2}{|c|}{ d'ouverture } \\
\hline nouveau thème & $\begin{array}{l}\text { Barnes (1995: 815), Hansen (1998a : 255), Brémond (2003 :73-74), Skattum (2012: } \\
\text { 210) }\end{array}$ \\
\hline prise de thème & Skattum $(2012: 210)$ \\
\hline nouvelle voix & « changement de point de vue » Barnes $(1995: 816)$ \\
\hline \multicolumn{2}{|c|}{ de continuation } \\
\hline sous-thème & $\begin{array}{l}\text { Winther (1985:89), Barnes (1995: 816), Brémond (2003: 75), Beeching (2007:81), } \\
\text { Beeching (2011:94), } \\
\text { « étapes ou séquences narratives » Skattum (2012:211) }\end{array}$ \\
\hline résultat & nouvelle fonction \\
\hline supplément & $\begin{array}{l}\text { « parenthèse explicative » Barnes }(1995: 815) \text {, «précision » Skattum }(2012: 212) \\
\text { « confirmation » Lee et al. }(2019: 2), \text { « explicatif [parenthetical] » Lee et al. }(2019: 2)\end{array}$ \\
\hline élément additif & nouvelle fonction \\
\hline \multicolumn{2}{|c|}{ d'autres fonctions textuelles } \\
\hline $\begin{array}{l}\text { reprise de } \\
\text { thème }\end{array}$ & Barnes (1995 : 815, 816), Brémond (2003: 73-75), Hansen (1998a : 255) \\
\hline formulation & $\begin{array}{l}\text { Barnes (1995: 814-815), Beeching (2007: 80), Beeching (2011: 95), Skattum (2012: } \\
\text { 213-14) } \\
\text { «reformulation » Eshkol-Taravella \& Grabar (2018: 4), Grabar \& Eshkol-Taravella } \\
(2016: 9) \\
\text { « hésitation » Winther }(1985: 89), \text { Lefeuvre }(2011 \mathrm{a}: 226), \text { Lefeuvre }(2011 \mathrm{~b}: 18) \\
\text { «gagner du temps » Crible (2017: 137), Beeching (2009: 220), Lee et al. (2019:2) }\end{array}$ \\
\hline \multicolumn{2}{|c|}{ Fonctions attitudinales } \\
\hline contraste & $\begin{array}{l}\text { « autocorrection » Skattum }(2012: 212), \text { « réparation » Beeching }(2009: 220) \\
\text { «opposition » Crible }(2017: 283)\end{array}$ \\
\hline résignation & $\begin{array}{l}\text { nouvelle fonction } \\
\text { «concession »Beeching }(2009: 220)\end{array}$ \\
\hline
\end{tabular}


Barnes (1995 : 815) propose la fonction de nouveau thème pour bon, et surtout pour la séquence bon alors, pas présente dans notre corpus, qui « est une expression par laquelle un locuteur peut prendre la parole et s'assurer de l'attention des autres participants ». Elle explique que bon marque la fin du discours précédent en même temps qu'il annonce l'ouverture de la conversation à « de nouvelles initiatives du locuteur et de nouvelles directions thématiques ». Donc, en plus d'identifier la fonction d'ouverture de bon, Barnes (1995: 815) lui attribue une fonction de clôture du discours précédent et d'appel à l'attention des interlocuteurs. Brémond (2003 : 74) est du même avis que Barnes (1995) que «bon permet à la fois de couper l'échange en cours et d'introduire une nouvelle séquence ainsi qu'un nouveau cadre énonciatif ». Skattum (2012:210) fournit un exemple de son corpus malien où un locuteur " clôt par bon la séance introductive (ça $v a$ ?) pour introduire un sujet de conversation ». Les trois auteures, tout en parlant de la fonction de bon d'amener un nouveau thème, soulignent sa fonction simultanée de terminer le discours précédent. Lefeuvre (2011b : 7), en citant Brémond (2002), constate brièvement que bon «permet au locuteur interviewer d'aborder un nouvel objet de commentaire ».

\subsubsection{Prise de thème}

Dans la catégorie d'ouverture se trouvent aussi les exemples de «prise de thème ». Ici l'interlocuteur amène le nouveau thème et le locuteur signale par bon son assentiment de le poursuivre. Waltereit (2007: 100), sans employer le terme " prise de thème », décrit cette fonction comme une sorte d' « acceptation », spécifiquement « l'expression de la volonté de poursuivre vers l'unité suivante». Skattum (2012: 210), tout comme Waltereit (2007), dit que bon "peut signaler que $\mathrm{B}$ accepte de répondre à $\mathrm{A}$ », mais elle signale que bon donne aussi au locuteur « un temps de réflexion pour se souvenir et organiser [son] récit». Cette fonction est illustrée dans notre corpus dans (6) où la locutrice dit « bon mon travail » pour accepter le sujet proposé par son interlocutrice et pour se donner le temps de préparer sa réponse.

6) D : [...] oui on n'a pas parlé de votre travail alors vous avez dit que $\mathrm{F}: \underline{\text { bon }}$ mon travail (AFS55, \#2-6)

Parfois bon est accompagné d'un autre élément comme dans (7). Il est possible que la locutrice (F) dise alors en écho du premier mot de la question de son interlocuteur.

7) D : alors si je voulais quitter Lille pour aller à la campagne il faudrait aller à quelle distance?

$\mathrm{F}:$ alors bon il y a [...] encore quelques campagnes qui restent dans le nord de la France, mais euh bon en général il faut parcourir quand même une vingtaine de kilomètres... (JFN35, \#14-1)

\subsubsection{Nouvelle voix}

La dernière fonction d'ouverture, que nous avons nommée « nouvelle voix », marque le début d'une citation dans le récit du locuteur. Dans l'exemple (8), la locutrice cite sa propre voix pour justifier sa décision de voir « un film nul» en Espagne. Ses mots «je me disais» indiquent clairement que bon marque le début d'une citation.

8) F : parce que [...] en Espagne le cinéma c'est moins cher qu'en France, donc $[\ldots]$ aller au cinéma même pour voir un film nul $[\ldots]$ $\mathrm{F}$ : je me disais bon, je vais entendre espagnol [...], c'est vrai j'ai regardé des émissions nulles (JFS25A \#6-4) 
Aucun auteur n'a proposé la fonction «nouvelle voix » pour bon, mais Hansen (1997: 176) a proposé cette fonction pour alors et Haileselassie $(2015: 139,142)$ a dit que voilà peut introduire une « citation hypothétique. » En outre, Barnes (1995: 817) maintient que ben et bon ben peuvent signaler le début du discours rapporté en citant Schiffrin (1985: 658), qui propose cette même fonction pour le MD well en anglais. Bien que Barnes (1995: 814) dise que cette fonction n'est jamais exprimée par bon tout seul, les huit occurrences de nouvelle voix dans notre corpus sont marquées par bon seul plutôt que par bon ben. Fleischmann et Yaguello (2004 : 135-136), dans leur analyse des fonctions des MD like en anglais et genre en français, emploient le terme "citative interprétative " ('interpretive quotative') pour cette fonction. Ce terme indique que le MD introduit une représentation des mots ou des pensées d'un tiers interprétés et reproduits par le locuteur. Ce terme est similaire au « dialogue construit » ('constructed dialogue') de Tannen (1986:313), terme qui souligne le fait que le locuteur prononce une version inexacte de la citation, plutôt qu'une citation textuelle, qui résume son sens général.

\subsection{Fonctions textuelles de continuation}

Les fonctions textuelles de bon regroupés sous la rubrique de continuation marquent une réorientation à l'intérieur d'un thème déjà établi. Plusieurs auteurs (Winther 1985: 89 ; Barnes 1995 : 816 ; Brémond 2003 : 75 ; Beeching 2007 : 81, 2011 : 94 ; Skattum 2012 : 211 ; Lee et al. $2019: 2$ ) ont signalé que bon peut marquer la progression du locuteur à travers les étapes de sa narration. Cependant, Skattum $(2012: 211)$ est la seule à fournir des exemples de bon dans une narration si celle-ci est définie comme une suite d'actions en ordre chronologique : «Dans le corpus malien, on est frappé tant par la fréquence de ces 'listings' chronologiques que par l'abondance de bon au sein de chacun ». Dans notre corpus, il n'y a pas d'exemples où bon ponctue les suites d'événements chronologiques, mais il y a trois exemples où et bon ou bon ben se trouve entre deux actions successives, comme dans (9) :

\section{9) F : Et puis il y avait un monsieur [...] qui a commencé à tituber. [...] il est pratiquement tombé dans le tramway et bon trois ou quatre personnes se sont précipitées pour l'aider [...]. (JFN26, \#13-2)}

Cependant, il est possible d'interpréter les étapes de la narration de façon thématique plutôt que chronologique comme « les étapes hiérarchisées de la progression du discours » (Brémond 2003 : 75). Cette observation par Brémond (2003) est similaire à la proposition faite par Auchlin (1981 : 146) que les MD indiquent le niveau de textualisation coordonnée ou subordonnée des énoncés (par exemple, «une justification est d'un niveau de textualisation inférieur à celui de l'acte dont le contenu fait l'objet de la justification » (Auchlin 1981 : 143-44)). Suivant cette définition, nous avons identifié deux fonctions de bon qui marquent la progression du discours entre des étapes thématiques.

\subsubsection{Sous-thème}

La première fonction de continuation textuelle est désignée comme «sous-thème » où bon marque le début d'une nouvelle unité thématique liée au thème central de la narration. Par exemple, la locutrice dans (10), qui parle de l'évolution économique de la France, introduit par bon la transition entre la domination du nord et la crise suivante.

10) F : pendant très très longtemps le nord a été un berceau industriel de de la France bon après il y a eu bien sûr une situation de crise parce que c'est surtout l'industrie textile qui fonctionnait (JFN35, \#14-1) 
Dans l'exemple (11) le locuteur dit bon pour marquer la réorientation vers l'endroit où il a fait sa coopération civile, un sous-thème de son récit sur cette expérience :

11) M : Donc c'était euh ça comptait pour une coopération civile [...] Donc j'ai préféré aller faire une coopération euh bon il s'est trouvé que c'était le Chili mais ça aurait pu être l'Afrique, ça aurait pu être l'Asie [...] (AHS65, \#12-1)

\subsubsection{Résultat}

La deuxième fonction de bon pour marquer la progression du discours est celle que nous désignons par « résultat ». La différence entre le sous-thème et le résultat est que le sousthème est une nouvelle unité thématique et le résultat est une résolution du thème en cours. Dans (12) la locutrice introduit le résultat de sa relation avec son ex-mari par bon.

12) F : Donc nous nous sommes installés dans cette maison pour cinq ans et puis la vie a fait que, ben ça fait seize ans.

$\mathrm{D}:$ Voilà.

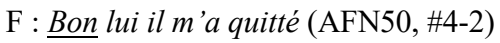

Dans (13) la locutrice introduit le résultat de la perte de vigueur des mots qu'on ne les entend plus par bon accompagné de plusieurs autres éléments :

13) F : après il y a des mots qui sont banalisés, et donc qu'on finit par employer puis après qui sont plus à la mode alors euh bon voilà on les entend plus. (AFN42, \#R8-4)

\subsubsection{Supplément}

Quand bon a la fonction textuelle de « supplément », il introduit l'insertion d'une explication ou commentaire bref, lié au thème actuel, qui fournit des informations supplémentaires. Barnes (1995: 815) décrit les renseignements introduits par bon comme « une parenthèse explicative qui interrompt [1']énoncé » tandis que Skattum $(2012: 212)$ les désigne comme une "précision ». Skattum (2012: 212) associe cet emploi à la digression proposée par Hansen (1995 : 30) où « [1]e locuteur interrompt provisoirement son discours en demandant à son interlocuteur d'accepter une digression apparente qui s'avérera pertinente pour la suite. » Une différence importante entre le supplément et le sous-thème dans notre schéma classificatoire est la brièveté des renseignements considérés comme un supplément et le retour au thème principal après cette parenthèse. Dans (14), par exemple, la locutrice interrompt sa description de la tournée des gouttes pour rassurer la deuxième auteure qu'on choisit prudemment les hôtes de ces visites nocturnes. L'importance de ce détail est soulignée par le fait que son fiancé $(\mathrm{M})$ le répète.

14) F : Alors ici on appelle ça [...] faire la tournée des gouttes [...]

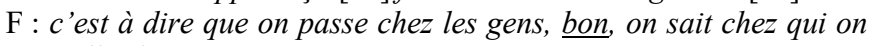
peut aller hein

$\mathrm{M}:$ qu'on connaît

$\mathrm{F}:$ et on va les réveiller à trois quatre heures du matin $[\ldots]$ et euh on vient chez eux et ils servent un alcool (JFS22, \#R13-7)

\subsection{4 Élément additif}

La dernière fonction de continuation dégagée dans notre corpus est l' « élément additif», une fonction de bon qui n'apparait pas dans les études antérieures. Dans ces contextes, bon se place au milieu d'une énumération pour signaler sa suite, fonction proposée aussi 
pour enfin (Buchi et Städtler 2008 : 6). Un exemple se trouve dans (15) où la locutrice insère bon au milieu de sa liste des attractions parisiennes.

15) F : Et donc euh, Paris oui, pour toute la diversité que ça, ça représente, [...] Les théâtres les musées, bon les cinémas, il y en a partout mais (AFN50 \#4-1)

\subsection{Autres fonctions textuelles}

Les deux dernières fonctions textuelles, qui ne sont classées ni comme ouverture ni comme continuation, sont la reprise d'un thème et la formulation.

\subsubsection{Reprise de thème}

Nous avons classé la fonction de bon comme « reprise de thème » quand il est suivi par une tentative du locuteur de reprendre un sujet discuté plus tôt dans la conversation. Brémond (2003 : 73) décrit cette fonction de bon comme une réintroduction ou recentrage sur le fil du discours, qu'elle définit comme « un point de focalisation mis en avant par les sujets et investi comme thématique et/ou tâche discursive dominante. » Cette fonction est illustrée dans (16) où la locutrice reprend la description de Béziers après la remarque de son interlocutrice que sa ville de résidence et Béziers sont de la même taille.

16) F : à Béziers

$\mathrm{D}:$ Oh, c'est à peu près la taille de la ville où on habite. [...] On a à peu près quatre-vingt-dix mille habitants

F : Bon. Euh, cette ville est, a un passé historique intéressant. Donc, eumm, nous avons une très belle cathédrale... (JFS32,\#11-3)

Les locuteurs de notre corpus se servent aussi des séquences avec bon, comme bon ben, et bon, euh bon, et et puis bon, pour marquer une reprise de thème. Dans (17) la locutrice dit puis après et ensuite bon ben pour reprendre la liste de ses villes de résidence après une parenthèse sur Bordeaux :

17) F : je n'ai pas du tout aimé Bordeaux. [...] Puis après, bon ben, après Bordeaux, ben je suis allée, [...] à Grenoble... (AFN50, \#4-1)

La fonction « reprise de thème » n'est pas très présente dans les études qui explorent les fonctions de bon. Barnes $(1995: 815,816)$ donne deux exemples où le locuteur exprime son intention de revenir sur le sujet du discours précédent en commençant par bon, mais elle classe le premier comme un nouveau thème et le deuxième comme un sousthème en disant qu'il «marque une transition entre les étapes ou segments de la narration ». Hansen (1998a : 255), de sa part, mentionne ce genre de reprise du thème comme une menace à la cohérence du discours non planifié, mais sans spécifier cette fonction pour bon.

\subsubsection{Formulation}

Nous avons regroupé sous la catégorie «formulation » toutes les occurrences où le locuteur dit bon pour formuler ou reformuler ses pensées. Pendant ces moments de formulation, bon est souvent accompagné de pauses, de répétitions, de faux-départs, ou de particules d'hésitation comme euh. Dans (18), par exemple, la locutrice hésite à décrire sa première rencontre avec son petit ami puisqu'il avait une autre copine à l'époque. Comme l'a expliqué Beeching (2011 : 95), un locuteur peut se douter de la meilleure façon de formuler son idée, surtout s'il s'agit d'une idée délicate qui peut provoquer un jugement ou réaction émotionnelle. 
18) D : Donc la première fois que vous l'avez vu c'était comment? $\mathrm{F}$ : Voilà c'était à un réveillon [...] donc euh euh il était bon il était pris mais ça a été donc le coup de foudre (JFS22, \#R13-5)

Dans (19) nous voyons clairement la tentative de formulation d'un locuteur qui dit euh avant et après bon :

19) D : mais pourquoi est-ce qu'on limite le nombre de thèses qu'on peut faire?

$\mathrm{M}$ : bé c'est, non mais au niveau financement $[\ldots]$

$\mathrm{M}$ : parce qu'il n'y a pas de financement, prendre des gens qui ont euh bon euh, plutôt une thèse commencée à [...] vingt-cinq ans, donc de vingt-cinq à vingt-huit et de pas payer les gens (JHS28, \#8-7)

Notre décision de regrouper la formulation et l'hésitation dans une seule catégorie suit les descriptions de cette catégorie par d'autres chercheurs. Barnes (1995 : 814-15) décrit bon comme « un simple marqueur d'hésitation ou de reformulation », une fonction qui s'ensuit logiquement de ses autres fonctions de mettre fin à un thème et d'en initier un autre. Beeching (2011 : 95) fait écho de cette idée en disant que bon peut marquer une reformulation syntaxique qui clôture le discours pour le redémarrer avec une nouvelle construction syntaxique. Elle marie les reformulations aux hésitations en parlant des « reformulations hésitatoires» ('hesitatory reformulations') (Beeching 2011: 95). Skattum (2012 : 213) associe bon intraphrastique, c'est-à-dire, à l'intérieur d'une phrase, avec l'hésitation et la réserve et elle associe la reformulation avec l'atténuation. Nous voyons donc comment une fonction textuelle peut être associée avec une fonction attitudinale. L'hésitation du locuteur, comme dans (18), peut signaler un désir de ne pas choquer ou offenser l'interlocuteur. Il faut pourtant remarquer que nous voyons aussi dans (18) que l'hésitation peut exister indépendamment de la reformulation. La locutrice hésite avant de dire « il était pris », mais elle ne reformule pas son expression de cette idée.

\subsection{Fonctions attitudinales de bon}

Les fonctions attitudinales que nous avons pu quantifier pour bon sont le contraste et la résignation.

\subsubsection{Contraste}

Le contraste est une réorientation attitudinale plutôt que textuelle. Au lieu de signaler une transition dans la structure du discours, le locuteur signale une réorientation de l'attitude ou les croyances des participants conversationnels. Nous avons identifié dans notre corpus trois sortes de contraste introduites par bon. La première est l'autocorrection où le locuteur corrige une idée qu'il vient d'exprimer. Dans l'exemple (20), le locuteur qualifie son accent de toulousain et ensuite il introduit par bon l'avis contraire qu'il n'est pas capable de le qualifier. Finalement, il dit mais bon pour se contredire de nouveau en repartant vers la première caractérisation de son accent comme toulousain.

20) M : Donc euh je qualifierais mon accent de accent toulousain bon je suis pas je suis pas capable de le, mais bon à priori oui c'est accent toulousain (AHS65, \#12-2)

Une deuxième sorte de contraste est l'expression d'une opposition entre deux idées qui ne constituent pas une autocorrection. Dans (21), la locutrice fait un contraste entre sa réaction à un commentaire sur ses cheveux par un Vietnamien et sa réaction si un Français disait la même chose. 
21) F : ...par exemple, moi j'avais un ami vietnamien, bon qui m'aimait beaucoup, qui m'avait dit [...] : "J'aime bien tes cheveux jaunes ». Bon, si quelqu'un en France me dit : "J'aime tes cheveux jaunes", je vais, je vais lui filer une paire de claques... (AFN55, \#10-2)

Un autre exemple de ce genre de contraste se trouve dans (22) où le locuteur implique un contraste entre les premiers jours de son voyage d'affaires avec ses collègues et l'expérience après un mois.

22) $\mathrm{M}$ : on se retrouve le soir à table à cinq ou six, c'est c'est vraiment euh sympa quoi, bon au bout d'un mois oui

$\mathrm{F}$ : que des garçons

M : c'est une vie d'hommes hein, [...] (JHS26, \#R13-12)

Le contraste peut représenter aussi un désaccord entre le locuteur et son interlocuteur. Un exemple de cette sorte de contraste se trouve dans (23). La locutrice corrige son interlocutrice qui fournit des noms des villes touristiques populaires qui ne correspondent pas à ses critères.

23) F : ...on partait tous faire des grands trucs, leur montrer la Côte

d'Azur, Marseille, on partait à Avignon, qui est moins loin [...]

$\mathrm{D}$ : Oui, et puis, il y a Arles, et Nîmes.

F : Il y a Arles, tout ça. Oui

D : Carcassonne

F : Mais, bon, euh, je veux dire, euh, Avignon Arles ce sont que des châteaux forts. Ce sont des châteaux. [...]

F: Mais, euh, il faut aller impérativement à Marseille hein. (AFS56A, \#3-2)

\subsubsection{Résignation}

La deuxième sorte de réorientation non textuelle est la «résignation». Cette fonction ressemble à l'indication de la « concession » notée par Beeching (2009:220). Ici le locuteur présente une difficulté mais ensuite réoriente son attitude vers l'acceptation de cette situation: « Il y a une difficulté, mais c'est bon; je l'accepte. » Dans l'exemple (24), la locutrice explique que la période de séparation entre elle et son copain a été difficile mais elle l'a acceptée. De toutes les fonctions que nous avons identifiées, c'est cette dernière qui rattache bon le plus à ses origines d'un adjectif qui exprime une évaluation positive.

24) F : Voilà donc ça a été un peu difficile la séparation mais bon maintenant [...] on est ensemble, donc c'est bon (JFS22, \#R13-5)

\section{Répartition des occurrences de bon et leur position}

\subsection{La répartition des occurrences de bon}

Cette section est consacrée à la fréquence des fonctions textuelles et attitudinales de bon décrites dans la section précédente et la possible corrélation entre ces fonctions et la place de bon dans le discours. Les fonctions des 227 occurrences de bon dans notre corpus sont réunies dans la première colonne à gauche du Tableau 2. On observe que les fonctions textuelles de bon sont bien plus fréquentes (66.5\%) que les fonctions attitudinales $(33.5 \%)$. Nous confirmons donc que bon sert souvent à réorienter la direction thématique du discours. Parmi les fonctions textuelles, bon marque le plus souvent la continuation 
$(38,3 \%)$, surtout un sous-thème (16\%), plutôt qu'un nouveau thème. Parmi les fonctions attitudinales, bon marque le contraste (24\%) plus souvent que la résignation ( $9 \%$ ).

Il est impossible de comparer nos résultats quantitatifs pour les fonctions de bon avec ceux des études antérieures. Quelques études qui ont compté les occurrences de bon dans un corpus ont organisé les occurrences selon la place de bon. Winther (1985: 85-89), par exemple, rend compte du nombre d'occurrences de bon comme constituant initial d'intervention (29), constituant unique d'intervention (8), et constituant inséré dans une intervention (12). Lefeuvre (2011a: 238) organise les occurrences de bon selon la structure syntaxique, par exemple, dans des unités syntaxiques autonomes verbales ou averbales ou à l'intérieur de la chaîne syntagmatique, schéma présenté aussi dans Lefeuvre (2011b). La quantification de bon par d'autres auteurs visait un autre but. Barnes (1995: 814) a trouvé que bon était moins fréquent dans son corpus que ben et enfin, tandis que Skattum (2012 : 207) a constaté que bon s'employait plus souvent au Mali qu'en Afrique francophone ou dans le monde francophone en général. Beeching (2007) a fait une étude sociolinguistique pour voir la corrélation entre l'emploi de bon et de tels facteurs que l'âge et le niveau d'éducation des locuteurs.

Tableau 2. La fréquence des fonctions de bon en général et selon la position

\begin{tabular}{|c|c|c|c|c|c|}
\hline & \multirow[t]{2}{*}{ TOTAL } & \multicolumn{4}{|c|}{ POSITION } \\
\hline & & $\begin{array}{l}\text { début } \\
\text { tour }\end{array}$ & $\begin{array}{l}\text { début } \\
\text { phrase }\end{array}$ & $\begin{array}{l}\text { milieu } \\
\text { phrase }\end{array}$ & fin \\
\hline $\begin{array}{l}\text { FONCTIONS } \\
\text { TEXTUELLES }\end{array}$ & $\begin{array}{l}66,5 \% \\
(151)\end{array}$ & $\begin{array}{l}19 \% \\
(29)\end{array}$ & $\begin{array}{l}30 \% \\
(45)\end{array}$ & $\begin{array}{l}49 \% \\
(74)\end{array}$ & $\begin{array}{l}2 \% \\
(3)\end{array}$ \\
\hline ouverture & $\begin{array}{l}11 \% \\
(25)\end{array}$ & $\begin{array}{l}36 \% \\
(9)\end{array}$ & $\begin{array}{l}8 \% \\
(2)\end{array}$ & $\begin{array}{l}52 \% \\
(13)\end{array}$ & $\begin{array}{l}4 \% \\
(1)\end{array}$ \\
\hline nouveau thème & $\begin{array}{l}7 \% \\
(17)\end{array}$ & $\begin{array}{l}53 \% \\
(9)\end{array}$ & $\begin{array}{l}6 \% \\
(1)\end{array}$ & $\begin{array}{l}35 \% \\
(6)\end{array}$ & $\begin{array}{l}6 \% \\
(1)\end{array}$ \\
\hline nouvelle voix & $\begin{array}{l}4 \% \\
(8)\end{array}$ & & $\begin{array}{l}12,5 \% \\
(1)\end{array}$ & $\begin{array}{l}87,5 \% \\
(7)\end{array}$ & \\
\hline continuation & $\begin{array}{l}38,3 \% \\
(87)\end{array}$ & $\begin{array}{l}15 \% \\
(13)\end{array}$ & $\begin{array}{l}41 \% \\
(36)\end{array}$ & $\begin{array}{l}43 \% \\
(37)\end{array}$ & $\begin{array}{l}1 \% \\
(1)\end{array}$ \\
\hline sous-thème & $\begin{array}{l}16 \% \\
(36)\end{array}$ & $\begin{array}{l}17 \% \\
(6)\end{array}$ & $\begin{array}{l}58 \% \\
(21) \\
\end{array}$ & $\begin{array}{l}25 \% \\
(9)\end{array}$ & \\
\hline résultat & $\begin{array}{l}11 \% \\
(24)\end{array}$ & $\begin{array}{l}4 \% \\
(1)\end{array}$ & $\begin{array}{l}33 \% \\
(8)\end{array}$ & $\begin{array}{l}63 \% \\
(15)\end{array}$ & \\
\hline supplément & $\begin{array}{l}12 \% \\
(27)\end{array}$ & $\begin{array}{l}22 \% \\
(6)\end{array}$ & $\begin{array}{l}26 \% \\
(7)\end{array}$ & $\begin{array}{l}48 \% \\
(13)\end{array}$ & $\begin{array}{l}4 \% \\
(1)\end{array}$ \\
\hline $\begin{array}{l}\text { autres fonctions } \\
\text { textuelles }\end{array}$ & $\begin{array}{l}17,2 \% \\
(39)\end{array}$ & $\begin{array}{l}18 \% \\
(7)\end{array}$ & $\begin{array}{l}18 \% \\
(7)\end{array}$ & $\begin{array}{l}61,5 \% \\
(24)\end{array}$ & $\begin{array}{l}2,5 \% \\
(1)\end{array}$ \\
\hline reprise de thème & $\begin{array}{l}6 \% \\
(14)\end{array}$ & $\begin{array}{l}43 \% \\
(6)\end{array}$ & $\begin{array}{l}43 \% \\
(6)\end{array}$ & $\begin{array}{l}14 \% \\
(2)\end{array}$ & \\
\hline formulation & $\begin{array}{l}11 \% \\
(25) \\
\end{array}$ & $\begin{array}{l}4 \% \\
(1)\end{array}$ & $\begin{array}{l}4 \% \\
(1) \\
\end{array}$ & $\begin{array}{l}88 \% \\
(22)\end{array}$ & $\begin{array}{l}4 \% \\
(1)\end{array}$ \\
\hline $\begin{array}{l}\text { FONCTIONS } \\
\text { ATTITUDINALES }\end{array}$ & $\begin{array}{l}33,5 \% \\
(76)\end{array}$ & $\begin{array}{l}18 \% \\
(14)\end{array}$ & $\begin{array}{l}37 \% \\
(28)\end{array}$ & $\begin{array}{l}34 \% \\
(26) \\
\end{array}$ & $\begin{array}{l}11 \% \\
(8)\end{array}$ \\
\hline contraste & $\begin{array}{l}24 \% \\
(55)\end{array}$ & $\begin{array}{l}18 \% \\
(10)\end{array}$ & $\begin{array}{l}49 \% \\
(27)\end{array}$ & $\begin{array}{l}31 \% \\
(17) \\
\end{array}$ & $\begin{array}{l}2 \% \\
(1)\end{array}$ \\
\hline résignation & $\begin{array}{l}9 \% \\
(21)\end{array}$ & $\begin{array}{l}19 \% \\
(4)\end{array}$ & $\begin{array}{l}5 \% \\
(1)\end{array}$ & $\begin{array}{l}43 \% \\
(9)\end{array}$ & $\begin{array}{l}33 \% \\
(7)\end{array}$ \\
\hline$\%$ du total $(\mathrm{N})$ & $\begin{array}{l}100 \% \\
(227)\end{array}$ & $\begin{array}{l}19 \% \\
(43)\end{array}$ & $\begin{array}{l}32 \% \\
(73)\end{array}$ & $\begin{array}{l}44 \% \\
(100)\end{array}$ & $\begin{array}{l}5 \% \\
(11)\end{array}$ \\
\hline
\end{tabular}

\subsection{La position de bon}

Inspirées par les études de Winther (1985:85-89), Hansen (1998b :234-47) et Skattum (2012 :210-13), qui ont organisé la présentation des fonctions de bon selon sa place dans le discours, nous avons voulu voir s'il y avait une corrélation entre la fonction de bon et 
sa place. Quelques chercheurs maintiennent que les MD se trouvent le plus souvent en début d'énoncé (Schiffrin 1987, Brinton 1996, Fraser 1996, Crible 2017 : 47-48), comme alors et donc (Hansen 1997 : 156, Degand 2014 : 153), tandis que d'autres attribuent plus de liberté positionnelle aux MD en disant qu'ils peuvent être placés au début, au milieu, ou à la fin d'une phrase (Connors 2016: 28, Degand et Fagard 2011 : 26-27, Hansen 1997 : 156). Gilbert (2019) a étudié la place de bon par rapport à la syntaxe des éléments qui l'entouraient dans le même corpus que pour cette étude. Elle a trouvé que bon se trouve le plus souvent au milieu d'un tour de parole entre deux structures syntaxiquement complètes.

Pour former une idée de la corrélation entre les fonctions de bon et sa position, nous avons exploré quatre positions dans le tour de parole ou la phrase : bon en début de tour de parole (comme dans (1) ci-dessus), en début de phrase mais à l'intérieur du tour de parole (comme dans (2) et (3)), au milieu d'une phrase (comme dans (8)), et en position finale. Il y avait si peu d'occurrences en position finale que nous avons confondu les 7 occurrences en fin de phrase (comme dans (24)), avec les 4 occurrences en fin de tour de parole (comme dans (25) ci-dessous). Il n'y avait pas d'occurrences où bon a été l'élément unique dans un tour.

\section{5) D : donc normalement les vaches laitières on ne les mange pas \\ $\mathrm{M}$ : c'est c'est une viande mauvaise, pas mauvaise mais $\underline{\text { bon }}$ (AHS55, \#R12-3)}

Les pourcentages dans le Tableau 2 ci-dessus montrent les positions les plus fréquentes pour les différentes fonctions de bon. En général, bon se trouve le plus souvent en milieu de phrase (44\%), mais les occurrences de bon avec une fonction textuelle (49\%) se trouvent plus souvent dans cette position que celles qui ont une fonction attitudinale (34\%). Les fonctions où bon apparait au milieu d'une phrase sont les fonctions textuelles de la formulation $(88 \%)$, la nouvelle voix $(87,5 \%)$, le résultat $(63 \%)$, et le supplément $(48 \%)$ et la fonction attitudinale de la résignation (43\%). Par contre, plus de la moitié des occurrences qui introduisent un nouveau thème se trouvent en début de tour de parole $(53 \%)$ et les occurrences avec la fonction d'une reprise de thème se trouvent soit en début de tour de parole (43\%) soit en début de phrase (43\%), ce qui est aussi la position la plus fréquent pour marquer un sous-thème $(58 \%)$. Le contraste aussi se trouve le plus souvent en début de phrase (49\%).

Il est remarquable que bon ne se trouve en position finale que très rarement $(5 \%)$; parmi les 11 occurrences dans cette position, 7 expriment la résignation. Dans le seul exemple de bon en fin de phrase classé comme nouveau thème, le locuteur s'apprête à introduire un nouveau thème, mais il ne le poursuit pas en disant simplement : " voyez ? bon" (AHS55, \#R12-2), et ensuite l'intervieweuse propose un nouveau sujet de conversation. Il est possible, par contre, qu'il ait dit bon pour clore son tour de parole.

Malgré les tendances que nous avons pu dégager entre la fonction pragmatique de bon et sa position dans le tour de parole ou la phrase, il est important de remarquer qu'il n'y a pas de corrélation exacte entre fonction et position. La place de bon dépend du moment où le locuteur ressent l'envie de réorienter la conversation ou exprimer l'acceptation. On peut donc conclure que la position de bon peut aider les chercheurs à organiser ses occurrences, comme l'ont fait Winther (1985 : 85-89), Hansen (1998b : 234-247), et Skattum (2012:210-13), mais il est préférable dans une étude des fonctions de bon de ne pas supposer à priori que la fonction de bon pourra être déterminée par sa position.

\section{La fonction de bon avec d'autres éléments}

Pour mieux cerner la contribution de bon dans le discours, nous avons identifié les éléments dans le corpus immédiatement avant ou après bon qui exprimaient une des fonctions 
identifiées pour bon ou une fonction similaire tels que mais pour le contraste, euh et ben pour l'hésitation qui peut accompagner une formulation ou reformulation, puis et parce que pour une suite ou un résultat, et oui et voilà pour l'acceptation. Parmi les séquences où un élément précède bon, la plus commune est mais bon (39), suivie par euh bon (25), et bon (12), (et) puis bon (10), parce que bon (8), et oui bon (6). Quant aux séquences avec des éléments après bon, on trouve bon ben (10), euh bon (9), bon mais (8), et bon voilà (6). Dans les cas de mais bon et euh bon, nous avons compté dans ces chiffres des séquences avec plus de deux éléments, comme mais euh bon, mais euh mais bon, mais enfin euh déjà bon, euh oui bon, et euh du fait bon.

Comme point de départ nous supposons que les deux éléments dans ces séquences retiennent chacun un sens individuel au lieu de former une nouvelle unité avec son propre sens. Dans les termes de Hansen (1998b : 233) pour ah bon, adoptés aussi par Waltereit (2007: 104) pour bon ben, les collocations ont un sens "sommatif» ou «compositionnel» plutôt que «combinatoire» ou « holistique». Selon cette approche, il est possible de comparer la séquence avec et sans bon pour mieux cerner la contribution sémantico-pragmatique de bon. Dans la séquence ah bon, par exemple, Hansen (1998b : 233) maintient que $a h$ exprime la surprise du locuteur à la réception des nouveaux renseignements et que bon exprime son acceptation provisoire de la vérité de ces renseignements. Waltereit (2007: 103) propose pour bon ben que le locuteur signale par bon qu'il « accepte la déclaration de son interlocuteur...; avec ben il la déclare en même temps non pertinente ». D'après Dostie (2012:119), c'est le deuxième élément dans ce type de séquence qui « appuie (ou insiste) sur les sens exprimés par les marqueurs-têtes auxquels ils sont joints ». Nous verrons si le premier élément dans les séquences dans notre corpus peut remplir à lui seul la fonction attribuée à la séquence complète et si bon ne fait que renforcer le sens du premier élément quand il se trouve à la fin d'une séquence ou s'il fait sa propre contribution au sens de la séquence.

\section{1 mais bon et bon mais}

Parmi les occurrences de mais bon, la séquence avec bon la plus fréquente de notre corpus, nous en avons classé 16 comme exprimant la résignation, comme la séquence dans (26). La conjonction adversative mais marque l'opposition à un inconvénient déjà exprimé et bon signale l'acceptation de cette situation et donc la résignation. Si la locutrice avait dit « un peu chaud donc euh mais » sans bon, elle n'aurait pas complété son idée. En effet, dans tous les exemples de la résignation dans le corpus (mais bon, donc bon, et bon ben), bon est accompagné d'un autre élément.

26) F : Et voilà donc euh, alors la vie ici, c'est très bien au niveau climat, quoiqu'en été il fasse

D : Ouais, [rires]

F : Un peu chaud, donc euh, mais bon. Moi je viens du d'un pays froid, j'ai un peu de mal en été. (AFN50, \#4-2)

La séquence mais bon signale un contraste plutôt que la résignation dans 22 de ses occurrences, dont une est présentée dans (27). La locutrice aurait pu exprimer le contraste en disant simplement « $\underline{\text { mais }}$ estomper les fautes », mais elle a choisi d'ajouter bon. Ici bon semble renforcer la réorientation contrastive exprimée par mais, comme l'avait proposé Dostie (2012 :119), en même temps qu'il exprime l'acceptation des réserves de l'interlocutrice sur les bénéfices de garder son accent.

27) F : Mais, bon, estomper les fautes pour que, pour que

$\mathrm{D}:$ qui empêchent la compréhension, qui prêtent à confusion

$\mathrm{F}$ : voilà, les personnes puissent comprendre ou voilà (JFS32, \#11-3) 
Quand mais suit bon dans la séquence bon mais, il paraît que bon introduit la réorientation et que mais introduit la proposition qui fournit le contenu du contraste. Encore une fois, le deuxième élément renforce le sens du premier puisque mais renforce l'opposition introduite par bon. Dans (28) on voit une réorientation textuelle vers un sousthème qui introduit en même temps un contraste entre le nombre d'étudiants dans le programme d'électronique du locuteur et le programme de Français Langue Étrangère de son amie.

28) $\mathrm{M}$ : on avait une promo de de vingt vingt-cinq étudiants en $D E A$, on va alors on va trouver une dizaine de thèses, bon mais chez eux ils sont combien? (JFS25, \#8-6)

Les exemples présentés dans cette section de bon avec mais montrent que bon peut exprimer à la fois la réorientation et l'acceptation. Quand mais bon exprime la résignation, la fonction de bon est d'exprimer surtout l'acceptation, mais quand cette séquence exprime le contraste, bon renforce la réorientation vers une idée opposée tout en acceptant ce qui a été dit auparavant. Dans la séquence bon mais, bon exprime surtout le début de la réorientation qui est complétée par la proposition qui commence par mais.

\section{2 euh bon et l'hésitation}

L'hésitation est un moyen d'adoucir la réponse du locuteur ou de signaler le début d'un sujet délicat, mais elle peut être le simple résultat de la difficulté du locuteur à formuler ses pensées, comme le disent Winther (1985), Hansen (1998b), et Beeching (2007, 2011). Dans les exemples de formulation où bon est accompagné de la particule d'hésitation euh, on veut savoir en examinant les exemples du corpus si bon renforce l'hésitation ou s'il a une autre fonction.

Considérons maintenant une des 25 occurrences de euh bon dans lesquelles bon est précédé, et parfois suivi, par la particule d'hésitation. Quand le locuteur dit bon après avoir hésité, comme dans (29), bon semble indiquer qu'il est prêt à redémarrer le fil de son discours. De cette façon, bon sert à marquer une réorientation vers la suite des idées après une hésitation. Le locuteur aurait pu bien faire sa phrase sans bon, mais il n'aurait pas indiqué son désir de poursuivre son intervention.

29) D: D'accord et comment est-ce que vous avez décidé d'aller étudier à Toulouse?

M: Ben parce que c'était ou Toulouse ou Montpellier ou ou Clermont-Ferrand

$D$ : D'accord ça c'était d'après la région...

$M:[. .$.$] administrativement on fait partie de la région Midi-Pyrénées$ mais en plus euh c'est euh bon c'est le c'est l'extrême bout de de la région Midi-Pyrénées c'est vrai que c'est aussi près de de Clermont-

Ferrand que de Montpellier, mais c'est quand même administrativement c'est la région Midi-Pyrénées (AHS65, \#12-1)

Quelquefois l'hésitation semble indiquer la réserve de la part du locuteur à exprimer une idée défavorable. Dans (30), le locuteur hésite à dire que les gens du nord soient moins accueillants que ceux du sud, mais il aurait pu exprimer cette idée sans bon puisque euh indique déjà son hésitation. Le rôle de bon ici est possiblement de donner un avertissement à l'interlocuteur et lui demander d'accepter ou d'excuser cette critique.

30) M : Paris c'est très grand pour accéder à son lieu de travail c'est des heures de déplacement, euh disons que ils ont pas beaucoup de moral et c'est là c'est où ils ont le taux de déprime euh le plus fort de France quoi sinon c'est quoi où il fait le plus mauvais quoi, et euh du fait bon 
ils sont un peu moins accueillants plus froids même avec les Français du sud... (JHS28, \#R10-1)

D'autres fois, quand la voyelle d'hésitation suit bon, comme dans (31) qui répète une partie de 23) ci-dessus, la locutrice dit mais bon pour introduire son désaccord avec l'interlocutrice et ensuite décide de l'atténuer en hésitant.

31) F : Mais, bon, euh, je veux dire, euh, Avignon Arles ce sont que des châteaux forts. Ce sont des châteaux. (AFS56,\#3-2)

Bon peut aussi se retrouver au milieu d'une série d'hésitations et de reformulations comme dans (32). Ici bon est peut-être une tentative échouée de redémarrage ou une demande de vérification et compréhension de la part de l'interlocutrice.

32) M: j'ai vu [aussitôt] au Chili quand je suis arrivé des jeunes américains jeunes français qui disaient ah euh tout ce qui vient de France tout ce qui vient des Etats-Unis tout ce qui vient d'Angleterre c'est parfait, et tout ce qu'il y a ici c'est nul

$\mathrm{D}$ : ouais ouais ouais

$\mathrm{M}$ : mais vous ne pouvez pas porter euh, bon, euh vous ne, évidemment d'abord vous allez provoquer une euh, une réaction de rejet (AHS65, \#12-3)

Il convient de dire que l'hésitation qui accompagne bon n'est pas toujours exprimée par euh. Dans (33), l'hésitation consiste de quatre éléments : ben, là, en fait et bon. Cette hésitation indique sans doute le contraste entre l'implication de l'interlocuteur qu'il pourra voyager pendant ses vacances et le fait qu'il les passe à travailler. L'hésitation semble inviter l'interlocutrice à puiser dans ses connaissances partagées pour se souvenir de ce fait. Il est possible aussi que bon est un marqueur de redémarrage, comme dans (29), qui marque la fin de l'hésitation et introduit l'explication du locuteur.

33) D : Quand est-ce que vous aurez des vacances euh?

$\mathrm{M}:$ Ben, là, en fait, bon, je suis en vacances normalement.

D : Mais, pas vraiment (JHS25, \#7-4)

\section{3 (et) puis bon}

L'adverbe puis pourrait indiquer une suite ou un résultat sans l'addition de bon. On peut supposer donc que dans la séquence puis bon c'est bon qui sert à demander l'assentiment des interlocuteurs. Dans (34), par exemple, la locutrice commence son tour de parole par puis bon pour affirmer l'importance de justifier l'investissement des parents, un avis partagé sans doute par les deux autres participants.

34) $\mathrm{M}$ : voilà et en fait euh on s'est mis à travailler deux mois avant [...] et puis finalement on a eu euh, on a eu tous les deux on a eu notre examen, bien quoi enfin

$\mathrm{D}$ : ouais?

$\mathrm{M}$ : ouais ouais ouais

$\mathrm{F}:$ puis bon quand les parents ont investi pendant deux ans (JFS22, \#R13-6)

Dans la collocation et puis bon dans (35), et puis serait suffisant pour indiquer la suite de la liste des endroits où le locuteur a habité. En ajoutant bon le locuteur fait appel aux connaissances partagées avec l'interlocutrice, comme il le dit bien. On pourrait considérer les connaissances partagées comme une catégorie spéciale d'acceptation : «Je sais que vous l'acceptez parce que vous le savez déjà. » 
33) M : et j'ai vécu en Suisse, je suis parti en Suisse pendant un an [...] et puis Montpellier, et puis bon le Maroc comme je vous ai dit l'année dernière pendant un an (JHN28, \#1-15)

\section{4 oui bon et bon voilà}

Dans les séquences oui bon et bon voilà, bon est accompagné d'un autre mot d'acceptation. Mais plutôt que renforcer l'acceptation, bon a une fonction de réorientation. Dans (36) le locuteur dit oui pour montrer son accord avec l'interlocutrice pendant qu'il cherche à formuler sa description des difficultés d'expression d'un de ses étudiants. Le bon suivant marque le redémarrage du discours après cette hésitation.

36) $\mathrm{M}$ : ce ce ce c'est pas que c'est, c'est pas que les phrases sont pas très jolies ou que le style est lourd. C'est que c'est que, soit ça veut rien dire, soit ça veut dire le contraire, hein?

$\mathrm{D}:$ D'accord oui donc c'est même pas clair

$\mathrm{M}:$ Là c'est à ce niveau-là c'est plus oui bon, on va mettre un mot plutôt qu'un autre parce que ça fait quatre fois qu'on le voit alors on va mettre un synonyme non non c'est pas ça, là c'est que j'ai du mal à comprendre (JHS28, \#8-4)

Dans (37) la locutrice dit bon pour faire le contraste entre sa situation actuelle et son avis qu'il faut la changer tandis que voilà semble indiquer la prise de conscience de la locutrice sur sa situation. Sans bon l'interlocutrice exprimerait cette nouvelle révélation mais sans marquer aussi clairement le contraste et la réorientation de son attitude envers sa situation.

37) F : je n'ai pas de statut, que je suis vacataire et j'ai quarante-trois ans et bon voilà ça peut pas durer comme ça (AFS43, \#18-1)

Cette première tentative d'analyser la fonction de bon avec d'autres éléments a montré que dans certains contextes la fonction de réorientation est mise en avant, comme dans la séquence mais bon qui exprime la résignation, et que dans d'autres c'est l'acceptation qui est privilégié, comme dans sa fonction d'indiquer le redémarrage du discours après une hésitation. Nous avons constaté en plus un élargissement de la notion d'acceptation pour inclure une demande de l'acceptation à l'interlocuteur.

\section{Conclusion}

À travers l'examen de 227 occurrences du marqueur discursif bon dans un corpus de français parlé, la présente étude a proposé une nouvelle organisation de ses fonctions pragmatiques. Nous avons trouvé que, d'une part, bon remplit de multiples fonctions textuelles de marquer l'ouverture $(11 \%)$, la continuation $(38 \%)$, ou la reprise $(6 \%)$ des unités de discours, ou la formulation ou la reformulation des idées du locuteur (11\%). D'autre part, il remplit aussi des fonctions attitudinales en marquant le contraste, la résignation, et l'atténuation du message du locuteur.

Dans la plupart des cas, le locuteur se sert de bon pour faire deux choses à la fois : accepter, ou au moins reconnaître, ce qui a précédé et indiquer une réorientation textuelle ou attitudinale. Ce changement de direction peut être plutôt grand comme l'introduction ou la reprise d'un (sous-)thème ou plutôt petit comme l'introduction d'un résultat, d'un supplément, ou d'une nouvelle voix. Ainsi, bon indique à l'interlocuteur que ce qui suit sera quelque peu différent de ce qui a précédé. La fonction de formulation peut marquer une nouvelle direction dans les cas où le locuteur veut redémarrer son discours pour mieux exprimer son idée, mais elle peut aussi marquer tout simplement les moments où le locuteur cherche ses mots. Quand bon marque un contraste, le locuteur change de direction en 
exprimant un avis différent de celui de son interlocuteur ou de son propre discours précédent ; ce contraste peut être adouci par l'emploi d'une séquence comprise de bon avec d'autres éléments. Quand le locuteur se sert d'un bon pour exprimer la résignation, il insiste surtout sur la fonction de bon d'exprimer l'acceptation plutôt qu'une réorientation. Nous proposons que bon est essentiellement un marqueur discursif d'acceptation et de réorientation.

Dans l'avenir nous aimerions poursuivre l'analyse de bon avec d'autres éléments qui expriment des fonctions similaires à celles proposées ici pour bon, comme la discussion dans la Section 5 ci-dessus, pour continuer à dégager les fonctions sémantico-pragmatiques qui lui sont propres. Il serait producteur aussi de comparer les emplois de bon avec ceux des autres MD soit dans un corpus soit dans un questionnaire auprès des locuteurs natifs. Une étude de l'emploi de différents MD, comme bon, ben, alors, enfin, dans des conversations naturelles et de l'interprétation de ces MD par des locuteurs natifs aideraient à raffiner davantage notre compréhension de leurs fonctions.

Nous tenons à remercier Jane Alexander Gilbert pour son aide avec la discussion des études antérieures et pour ses conseils sur la position syntaxique de bon ainsi que nos deux relecteurs pour leurs commentaires fort utiles et très appréciés. Nous exprimons aussi notre vive gratitude à nos participants de Montpellier et Rognes pour leur collaboration, hospitalité, et générosité.

\section{Références bibliographiques}

Auchlin, A. (1981). Mais, heu, pis bon, ben alors voilà, quoi : Marqueurs de structuration de la conversation et complétude. Cahiers de linguistique française, 2, 141-60.

Barnes, B. (1995). Discourse Particles in French Conversation : (eh)ben, bon, and enfin. The French Review, 68, 813-21.

Beeching, K. (2007). La co-variation des marqueurs discursifs bon, c'est-à-dire, enfin, hein, quand même, quoi et si vous voulez : une question d'identité ?. Langue française, 154, 78-93.

Beeching, K. (2009). Sociolinguistic factors and the pragmaticalization of bon in contemporary spoken French. Dans K. Beeching, N. Armstrong, et F. Gadet (éds.), Sociolinguistic variation in contemporary French, 217-29. Amsterdam: John Benjamins.

Beeching, K. (2011). The translation equivalence of bon, enfin, well, and I mean. Revue de la linguistique française appliquée, 16, 91-105.

Brémond, C. (2002). Les petites marques du discours : le cas du marqueur métadiscursif bon en français. Thèse de doctorat. Université d'Aix-Marseille I.

Brémond, C. (2003). Bon, moteur d'action, moteur du discours. Travaux Interdisciplinaires $d u$ Laboratoire Parole et Langage d'Aix-en-Provence (TIPA) Laboratoire Parole et Langage, $22,65-84$.

Brinton, L. (1996). Pragmatic Markers in English : Grammaticalization and Discourse Functions. New York: Mouton de Gruyter.

Buchi, E. et T. Städtler. (2008). La pragmaticalisation de l'adverbe enfin du point de vue des romanistes («Enfin, de celui des francisants qui conçoivent leur recherche dans le cadre de la linguistique romane »). Congrès Mondial de Linguistique Française, 159-171.

Connors, M. D. (2016). The Pragmatic Particles enfin and écoute in French Film and TV Dialogue. Thèse de doctorat. University of Exeter.

Crible, L. (2017). Discourse Markers and (Dis)fluency across Registers: A Contrastive UsageBased Study in English and French. Thèse de doctorat. Université Catholique de Louvain.

Dajko, N. et K. Carmichael. (2014). But qui c'est la différence? Discourse markers in Louisiana French. Language in Society, 43, 159-83. 
Degand, L. (2014). 'So very fast very fast then' Discourse markers at left and right periphery in spoken French. Dans K. Beeching et U. Detges (éds.), Discourse Functions at the Left and Right Periphery: Crosslinguistic Investigations of Language Use and Language Change. Brill : Leiden, 151-78.

Degand, L. et B. Fagard. (2011). Alors between discourse and grammar: the role of syntactic position. Functions of Language, 18, 19-56.

Dostie, G. (2009). Discourse markers and regional variation in French: A lexico-semantic approach. Dans K. Beeching, N. Armstrong, et F. Gadet (éds.), Sociolinguistic variation in contemporary French, 201-14. Amsterdam: John Benjamins, 201-14.

Dostie, G. (2012). Ben en tant que collocatif discursif. Travaux de linguistique, 65, 102-22.

Eshkol-Taravella, I. et N. Grabar. (2018). Reformulations avec et sans marqueurs : étude de trois entretiens de l'oral. Dans F. Neveu, B. Harmegnies, L. Hriba et S. Prévost (éds.), $\sigma^{e}$ Congrès Mondial de Linguistique Française 2018, 46, 1-14.

Fleischmann, S. et M. Yaguello. (2004). Discourse Markers across Languages : Evidence from English and French. Dans C. L. Moder et A. Marinovic-Zic (éds.), Discourse Across Languages and Cultures. Amsterdam : John Benjamins, 129-47.

Frank-Job, B. (2005). A dynamic-interactional approach to discourse markers. Dans K. Fischer (éd.), Approaches to Discourse Markers, 395-413. Oxford : Elsevier.

Fraser, B. (1996). Pragmatic markers. Pragmatics, 6, 167-90.

Gilbert, J. A. (2019). The syntactic environment of the French discourse marker bon. Universite de Géorgie. Thèse de maitrise inédite.

Grabar, N. et I. Eshkol-Taravella. (2016). Prédiction automatique de fonctions pragmatiques dans les reformulations. TALN 2016: Traitement Automatique des Langues Naturelles, 1-14. Paris, France.

Hansen, M-B. (1995). Marqueurs métadiscursifs en français parlé : l'exemple de bon et de ben. Le Français Moderne, 63.1, 21-41.

Hansen, M-B. (1997). Alors and donc in spoken French: A reanalysis. Journal of Pragmatics, 28, $153-87$.

Hansen, M-B. (1998a). The semantic status of discourse markers. Lingua, 104, 235-260.

Hansen, M-B. (1998b). The Function of Discourse Particles. Amsterdam : John Benjamins.

Haileselassie, A. (2015). Voilà, An Orientation Shift Marker in Modern French Discourse: A Conversation Analytic Prescriptive. Thèse de doctorat. University of Illinois at UrbanaChampaign.

Heine, B. (2013). On discourse markers : Grammaticalization, pragmaticalization, or something else ? Linguistics, 51(6), 1205-47.

Jayez, J. (2004). Bon : le mot de la fin. Communication à l'Université de Genève. $<$ http://jjayez.pagesperso-orange.fr/doc/bon.pdf $>$

Lee, L., K. Bartkova, D. Jouvet, M. Dargnat, and Y. Keromnes. 2019. Can prosody meet pragmatics? Case of discourse markers in French. ICPhS2019 International Congress of Phonetic Sciences. Melbourne, Australia.

Lefeuvre, F. (2011a). Bon et quoi à l'oral: marqueurs d'ouverture et de fermeture d'unités syntaxiques à l'oral. Linx : Revue des linguistes de l'université Paris X Nanterre, 64-65, 223240.

Lefeuvre, F. (2011b). Bon dans le discours oral : une unité autonome? $<$ https://halshs.archives-ouvertes.fr/halshs-00797188/document $>$

Schiffrin, D. (1987). Discourse Markers. Cambridge: Cambridge University Press. 
Skattum, I. (2012). Bon, marqueur discursif en français parlé au Mali. Le Français en Afrique, 27, 201-17.

Tannen, D. (1986). Introducing constructed dialogue in Greek and American conversational and literary narrative. Dans F. Coulmas (éd.), Direct and Indirect Speech, 311-32. Berlin : Mouton.

Waltereit, R. (2007). À propos de la genèse diachronique des combinaisons de marqueurs : L'exemple de bon ben et enfin bref. Langue française, 154, 94-109.

Winther, A. (1985). Bon (bien, très bien) : ponctuation discursive et ponctuation métadiscursive. Langue Française, 65, 80-91.

\footnotetext{
${ }^{1}$ Dans les extraits du corpus, un M ou F marque le tour de parole d'un locuteur français ou une locutrice française, respectivement, et D marque une intervention de l'intervieweuse. Chaque extrait est suivi par l'identifiant du locuteur, décrit dans la section 2, ainsi que le numéro et la tranche de l'enregistrement dans le corpus.
} 